\title{
ROBUST NOVEL HIGH-ORDER ACCURATE NUMERICAL METHODS FOR SINGULARLY PERTURBED CONVECTION-DIFFUSION PROBLEMS $^{1}$
}

\author{
G.I. SHISHKIN \\ Institute of Mathematics and Mechanics \\ Russian Academy of Sciences, Ekaterinburg 620219, GSP-384, Russia \\ E-mail: shishkin@imm.uran.ru
}

Received September 1, 2005; revised October 15, 2005

This paper is dedicated to N.S. Bakhvalov

\begin{abstract}
For singularly perturbed boundary value problems, numerical methods convergent $\varepsilon$-uniformly have the low accuracy. So, for parabolic convection-diffusion problem the order of convergence does not exceed one even if the problem data are sufficiently smooth. However, already for piecewise smooth initial data this order is not higher than $\frac{1}{2}$. For problems of such type, using newly developed methods such as the method based on the asymptotic expansion technique and the method of the additive splitting of singularities, we construct $\varepsilon$ uniformly convergent schemes with improved order of accuracy.
\end{abstract}

Key words: singular perturbation, boundary layer, parabolic convection-diffusion equation, difference scheme, parameter-uniform convergence, high-order accuracy

\section{Introduction}

Solutions of singularly perturbed convection-diffusion problems have boundary layers controlled by a perturbation parameter $\varepsilon$. This parameter multiplies the highest space derivatives in equations and is related to the thickness of the boundary layer. It is well known that standard numerical methods give large errors in discrete solutions (comparable with the exact solution) when $\varepsilon$ becomes small. That is why robust numerical methods, i.e., methods whose errors are independent of the parameter $\varepsilon$ (or in short, $\varepsilon$-uniform methods), are very important. At present robust numerical methods have been developed for various problems with boundary layers. However, the order

\footnotetext{
${ }^{1}$ This research was supported by the Russian Foundation for Basic Research under grants No. 04-01-00578, 04-01-89007-NWO_a, by the Netherlands Research Organisation NWO under grant No. 047.016.008 and by the Boole Centre for Research in Informatics at the National University of Ireland, Cork.
} 
of accuracy for such $\varepsilon$-uniform special numerical methods does not exceed one for convection-diffusion equations in the maximum norm (see, e.g., $[2,12,13,18]$ and also the bibliography therein). The low rate of $\varepsilon$-uniform convergence is a significant restriction to use such methods in practice.

Thus, the development of $\varepsilon$-uniformly convergent numerical methods of highorder accuracy is sufficiently actual task for singularly perturbed problems. We mention existing techniques used when constructing $\varepsilon$-uniformly convergent finite difference schemes with improved accuracy such as well elaborated:

(a) the defect correction method,

(b) the Richardson extrapolation method,

and anew created

(c) the method based on the asymptotic expansion technique,

(d) the method of the additive splitting of singularities.

Methods based on approaches (a) and (b) are discussed in [3, 4, 7, 6, 8, 9] and $[15,20,25]$; approaches (c) and (d) are given in [21, 22, 24] and [10, 19, 23], respectively, see the references therein. These approaches, which use the technique based on piecewise uniform meshes condensing in the layer region, allow us to construct effective numerical methods for a sufficiently wide class of boundary value problems to elliptic and parabolic equations whose solutions exhibit layer phenomena. Originally, condensing mesh technique for singularly perturbed problems was invented by N.S. Bakhvalov in 1969 [1], and was developed in subsequent publications (see, e.g., $[2,12,18]$ and also the bibliography therein).

In the present paper, in order to demonstrate the techniques (c) and (d), we focus our attention on singularly perturbed parabolic convection-diffusion equations with smooth (Sections 2-4) and piecewise smooth initial data (Sections 5-8).

\section{Problem with Smooth Data. Aim of Research}

On the set $\bar{G}$

$$
\bar{G}=G \bigcup S, \quad G=D \times(0, T],
$$

where $D=(0, d)$, we consider the boundary value problem for the parabolic equation

$$
L u(x, t)=f(x, t), \quad(x, t) \in G, \quad u(x, t)=\varphi(x, t), \quad(x, t) \in S .
$$

Here

$$
L \equiv \varepsilon a(x, t) \frac{\partial^{2}}{\partial x^{2}}+b(x, t) \frac{\partial}{\partial x}-c(x, t)-p(x, t) \frac{\partial}{\partial t}, \quad(x, t) \in G,
$$

the functions $a(x, t), b(x, t), c(x, t), p(x, t), f(x, t)$ and $\varphi(x, t)$ are assumed to be sufficiently smooth on $\bar{G}$ and $S$, respectively, moreover ${ }^{1}$

\footnotetext{
1 Throughout this paper, $M, M_{i}$ (or $m$ ) denote sufficiently large (small) positive constants that do not depend on $\varepsilon$ and on the discretization parameters.
} 


$$
\begin{aligned}
& a_{0} \leq a(x, t) \leq a^{0}, \quad b_{0} \leq b(x, t) \leq b^{0}, \quad c_{0} \leq c(x, t) \leq c^{0}, \quad p_{0} \leq p(x, t) \leq p^{0} \\
& |f(x, t)| \leq M, \quad(x, t) \in \bar{G} ; \quad|\varphi(x, t)| \leq M, \quad(x, t) \in S ; \quad a_{0}, b_{0}, c_{0}, p_{0}>0
\end{aligned}
$$

the perturbation parameter $\varepsilon$ takes arbitrary values in $(0,1]$.

Assume that the data of problem (2.2), (2.1) on the set of corner points $S_{*}=$ $S_{0} \cap \bar{S}^{L}$ satisfy the compatibility conditions which ensure the required smoothness of the solution on $\bar{G}$ (see, e.g., [11]). Here $S=S_{0} \cup S^{L}, S_{0}$ and $S^{L}$ are the lower and lateral boundaries; $S_{0}=\bar{S}_{0}$.

For small $\varepsilon$, a regular boundary layer appears in a neighbourhood of the set

$$
S^{l}=\{(x, t): x=0,0<t \leq T\} .
$$

Here $S^{l}$ and $S^{r}$ are the left and right parts of the lateral boundary: $S^{L}=S^{l} \cup S^{r}$.

Let us give an $\varepsilon$-uniformly convergent finite difference scheme constructed by a classical approximation of problem (2.2), (2.1). On the set $\bar{G}$ we introduce the rectangular mesh

$$
\bar{G}_{h}=\bar{\omega} \times \bar{\omega}_{0}
$$

where $\bar{\omega}$ and $\bar{\omega}_{0}$ are arbitrary, generally speaking, non-uniform meshes on the segments $[0, d]$ and $[0, T]$, respectively. Let

$$
\begin{aligned}
& h^{i}=x^{i+1}-x^{i}, \quad x^{i}, x^{i+1} \in \bar{\omega}, \quad h=\max _{i} h^{i}, \\
& h_{t}^{k}=t^{k+1}-t^{k}, \quad t^{k}, t^{k+1} \in \bar{\omega}_{0}, \quad h_{t}=\max _{k} h_{t}^{k} .
\end{aligned}
$$

Assume that $h \leq M N^{-1}, h_{t} \leq M N_{0}^{-1}$, where $N+1$ and $N_{0}+1$ are the number of nodes in the meshes $\bar{\omega}$ and $\bar{\omega}_{0}$, respectively.

Problem (2.2), (2.1) is approximated by the finite difference scheme [14]

$$
\Lambda z(x, t)=f(x, t), \quad(x, t) \in G_{h}, \quad z(x, t)=\varphi(x, t), \quad(x, t) \in S_{h} .
$$

Here $G_{h}=G \bigcap \bar{G}_{h}, S_{h}=S \bigcap \bar{G}$,

$$
\Lambda \equiv \varepsilon a(x, t) \delta_{\bar{x} \widehat{x}}+b(x, t) \delta_{x}-c(x, t)-p(x, t) \delta_{\bar{t}}, \quad(x, t) \in G_{h},
$$

$\delta_{\bar{x} \widehat{x}} z(x, t)$ is the second central difference derivative on a nonuniform mesh,

$$
\delta_{\bar{x} \widehat{x}} z(x, t)=2\left(h^{i}+h^{i-1}\right)^{-1}\left[\delta_{x} z(x, t)-\delta_{\bar{x}} z(x, t)\right], \quad(x, t)=\left(x^{i}, t\right) \in G_{h} ;
$$

$\delta_{x} z(x, t)$ and $\delta_{\bar{x}} z(x, t)$ are the first (forward and backward) derivatives.

Scheme (2.5), (2.4) is monotone [14] $\varepsilon$-uniformly. In the case of uniform meshes in both variables

$$
\bar{G}_{h}=\bar{\omega} \times \bar{\omega}_{0}
$$

using the maximum principle, we obtain the estimate

$$
|u(x, t)-z(x, t)| \leq M\left[\left(\varepsilon+N^{-1}\right)^{-1} N^{-1}+N_{0}^{-1}\right], \quad(x, t) \in \bar{G}_{h},
$$

i.e. the scheme converges as $N^{-1} \ll \varepsilon, N, N_{0} \rightarrow \infty$. 
We now construct the base scheme that converges $\varepsilon$-uniformly (see, e.g., [6, 7, 8, 18]). On the set $\bar{G}$ we introduce the mesh

$$
\bar{G}_{h}=\bar{\omega}^{*} \times \bar{\omega}_{0},
$$

where $\bar{\omega}_{0}=\bar{\omega}_{0(2.6)}, \bar{\omega}^{*}$ is a piecewise uniform mesh constructed as follows. The segment $[0, d]$ is divided into two parts $[0, \sigma]$ and $[\sigma, d]$, where the step-sizes are constant and equal to $h^{(1)}=2 \sigma N^{-1}$ and $h^{(2)}=2(d-\sigma) N^{-1}$, respectively. The parameter $\sigma$ is defined by

$$
\sigma=\sigma(\varepsilon, N, l)=\min \left[2^{-1} d, l m^{-1} \varepsilon \ln N\right],
$$

where $m$ is an arbitrary number from $\left(0, m_{0}\right), m_{0}=\min _{\bar{G}}\left[a^{-1}(x, t) b(x, t)\right]$. Here

$$
l=1 .
$$

In other piecewise-uniform meshes this parameter will be chosen. Thus the meshes $\bar{\omega}^{*}$ and $\bar{G}_{h}=\bar{G}_{h}(l=1)$ are constructed.

For solutions of scheme (2.5), (2.8) we obtain the $\varepsilon$-uniform estimate

$$
|u(x, t)-z(x, t)| \leq M\left[N^{-1} \ln N+N_{0}^{-1}\right], \quad(x, t) \in \bar{G}_{h} .
$$

Theorem 1. Let the solution $u(x, t)$ of problem (2.2), (2.1) satisfies the estimates from Theorem 2, where $K=4$. Then the difference scheme (2.5), (2.8) (scheme (2.5), (2.6)) converges $\varepsilon$-uniformly (for fixed values of the parameter $\varepsilon$ ). The numerical solutions satisfy estimates (2.7) and (2.9).

For the boundary value problem (2.2), (2.1), our aim is to develop a finite difference scheme convergent $\varepsilon$-uniformly with accuracy higher than the first order, using the technique based on asymptotic constructs.

\section{A Priori Estimates and the Asymptotic Construction for Problem (2.2), (2.1)}

Here we give a priori estimates of the solutions and derivatives for problem (2.2), (2.1); the derivation of these estimates is similar to that in $[6,7,8,18]$.

We represent the solution of the problem as the sum of functions

$$
u(x, t)=U(x, t)+V(x, t), \quad(x, t) \in \bar{G},
$$

where $U(x, t)$ and $V(x, t)$ are the regular and singular parts of the solution. The function $U(x, t),(x, t) \in \bar{G}$ is the restriction, to $\bar{G}$, of the function $U^{e}(x, t),(x, t) \in$ $\bar{G}^{e}$, which is a solution of the "extended" problem obtained by extension of problem (2.2), (2.1) beyond the left boundary $S^{l}$, so that $\bar{G} \subset \bar{G}^{e}$, with retention of properties similar to (2.3). The function $V(x, t),(x, t) \in \bar{G}$, is the solution of the problem

$$
\left\{\begin{array}{l}
L_{(2.2)} V(x, t)=0, \quad(x, t) \in G, \\
V(x, t)=\varphi(x, t)-U(x, t), \quad(x, t) \in S .
\end{array}\right.
$$


For simplicity, besides sufficient smoothness of the data in problem (2.2), (2.1), we assume that

$$
\frac{\partial^{k}}{\partial x^{k}} \varphi(x, t), \frac{\partial^{k_{0}}}{\partial t^{k_{0}}} \varphi(x, t), \frac{\partial^{k+k_{0}}}{\partial x^{k} \partial t^{k_{0}}} f(x, t)=0, \quad(x, t) \in S_{*}, k, k_{0} \leq l,
$$

where $l=3 n+2, n \geq 1$. In this case

$$
u, U, V \in C^{l^{1}, l^{1}}(\bar{G}), \quad l^{1}=n+1+\alpha, \quad \alpha \in(0,1) ;
$$

for $U(x, t)$ and $V(x, t)$ we obtain the estimates (a technique can be found in [5])

$$
\begin{aligned}
&\left|\frac{\partial^{k+k_{0}}}{\partial x^{k} \partial t^{k_{0}}} U(x, t)\right| \leq M\left[1+\varepsilon^{n+1-k-k_{0}}\right], \\
&\left|\frac{\partial^{k+k_{0}}}{\partial x^{k} \partial t^{k_{0}}} V(x, t)\right| \leq M \varepsilon^{-k} \exp \left(-m \varepsilon^{-1} x\right), \quad(x, t) \in \bar{G}, \quad k+k_{0} \leq K,
\end{aligned}
$$

where $m$ is any number from the interval $\left(0, m_{0}\right), m_{0}=m_{0(2.8)} ; K=n+1$.

Theorem 2. Let the data of the boundary value problem (2.2), (2.1) satisfy the condition $a, b, c, p \in C^{l, l}(\bar{G}), f \in C^{l, l,}(\bar{G}), \varphi \in C^{l, l}(\bar{G}), l=3 n+2, n \geq 1$, and also condition (3.2). Then the solution of the problem and its components in representation (3.1) satisfy estimates (3.3), where $K=n+1$.

We now give the formulations of problem for main terms in the asymptotics of the solution that are considered in a neighbourhood of the boundary layer and outside it. We will use these problem formulations when constructing schemes for small values of the parameter. The set $\bar{G}$ can be represented in the form of a sum of two sets

$$
\begin{aligned}
& \bar{G}=\bar{G}^{(1)} \cup \bar{G}^{(2)}, \quad S^{(k)}=\bar{G}^{(k)} \backslash G^{(k)}, \quad k=1,2 ; \\
& G^{(1)}=(0, \sigma) \times(0, T], \quad G^{(2)}=[\sigma, d) \times(0, T] .
\end{aligned}
$$

The condition imposed on the parameter $\sigma$ is given below (in (3.6a)).

Let us introduce the function $u^{2}(x, t),(x, t) \in \bar{G}^{(2)}$, i.e., two first terms in the outer asymptotic expansion of the solution of problem (2.2), (2.1). We assume

$$
u^{2}(x, t)=u_{0}^{2}(x, t)+\varepsilon u_{1}^{2}(x, t), \quad(x, t) \in \bar{G}^{(2)} .
$$

The components $u_{i}^{2}(x, t)$ can be found by solving the problems

$$
\begin{gathered}
\left\{\begin{array}{l}
L^{(1)} u_{0}^{2}(x, t)=f(x, t), \quad(x, t) \in G^{(2)}, \\
u_{0}^{2}(x, t)=\varphi(x, t), \quad(x, t) \in S^{(2)},
\end{array}\right. \\
\left\{\begin{array}{l}
L^{(1)} u_{1}^{2}(x, t)=-a(x, t) \frac{\partial^{2}}{\partial x^{2}} u_{0}^{2}(x, t), \quad(x, t) \in G^{(2)}, \\
u_{1}^{2}(x, t)=0, \quad(x, t) \in S^{(2)},
\end{array}\right.
\end{gathered}
$$

where 


$$
L^{(1)} u(x, t) \equiv\left\{b(x, t) \frac{\partial}{\partial x}-c(x, t)-p(x, t) \frac{\partial}{\partial t}\right\} u(x, t), \quad(x, t) \in G^{(2)} .
$$

We say that the function $u^{2}(x, t),(x, t) \in \bar{G}^{(2)}$, is the solution of problem (3.5a;3.5b). Then we find the function $u^{1}(x, t),(x, t) \in \bar{G}^{(1)}$ by solving the problem

$$
\begin{aligned}
& L_{(2.2)} u^{1}(x, t)=f(x, t), \quad(x, t) \in G^{(1)}, \\
& u^{1}(x, t)=\left\{\begin{array}{l}
\varphi(x, t), \quad(x, t) \in S^{(1)} \bigcap S, \\
u^{2}(x, t),(x, t) \in S^{(1)} \backslash S .
\end{array}\right.
\end{aligned}
$$

The function $u^{1}(x, t)$ is a solution of the parabolic equation, but in a small (in $x$ ) subdomain.

Using asymptotic expansions of the components in representation (3.1) of the solution $u(x, t)$, provided that

$$
\sigma=\sigma(\varepsilon, \delta)=\min \left(2^{-1} d, q m^{-1} \varepsilon \ln (1 / \delta)\right),
$$

where $q \geq 1, m=m_{(3.3)}$ and $\delta$ is a sufficiently small number, we find the estimate

$$
\left|u(x, t)-u^{k}(x, t)\right| \leq M\left(\varepsilon^{2}+\delta^{q}\right), \quad(x, t) \in \bar{G}^{(k)}, \quad k=1,2 .
$$

Theorem 3. Let the hypothesis of Theorem 2 for $l=4$ be fulfilled. Then the functions $u^{k}(x, t),(x, t) \in \bar{G}^{(k)}, k=1,2$, satisfy estimate (3.6b) under condition (3.6a).

Remark 1. When the domain $\bar{G}$ is subdivided into subdomains (3.4), (3.6a) for $\delta \approx \varepsilon$, $q \geq 2$, the solutions of subproblems (3.5a; $3.5 \mathrm{~b}),(3.5 \mathrm{c})$ define on the subdomains $\bar{G}^{(k)}$ the functions $u^{k}(x, t), k=1,2$, which approximate the solution of the boundary value problem $(2.2),(2.1)$ with accuracy $\mathcal{O}\left(\varepsilon^{2}\right)$.

\section{Schemes with Improved Accuracy for Problem (2.2), (2.1)}

\subsection{Scheme with improved convergence for finite $\varepsilon$}

Here we give an $\varepsilon$-uniformly convergent difference scheme having (unlike scheme (2.5), (2.8)) the rate of $\varepsilon$-uniform convergence of order close to two in $x$, for not too small values of the parameter $\varepsilon$, and of the second order in $t$. The finite difference scheme is constructed by approximation of problem (2.2), (2.1) using the first central difference derivatives in $x$ and the defect correction for the implicit difference derivative in $t$.

On the set $\bar{G}$, we introduce the family of meshes

$$
\bar{G}_{h}=\bar{G}_{h}^{s} \equiv \bar{\omega}^{s} \times \bar{\omega}_{0},
$$

where $\bar{\omega}_{0}=\bar{\omega}_{0(2.8)}, \bar{\omega}^{s}$ is a piecewise uniform mesh on $\bar{D} ; \bar{\omega}^{s}=\bar{\omega}_{(2.8)}^{*}(\sigma)$ under the condition 


$$
\sigma=\sigma(\varepsilon, N, l=2)=\min \left(2^{-1} d, 2 m^{-1} \varepsilon \ln N\right), \quad m=m_{(3.3)} .
$$

On the mesh $\bar{G}_{h}$ we consider the difference scheme

$$
\left\{\begin{array}{l}
\Lambda^{(2)} z(x, t)=f(x, t), \quad(x, t) \in G_{h}, \\
z(x, t)=\varphi(x, t), \quad(x, t) \in S_{h} .
\end{array}\right.
$$

Here

$$
\begin{aligned}
& \Lambda^{(2)} z(x, t) \equiv\left\{\Lambda_{2}+\Lambda_{1}\right\} z(x, t), \\
& \Lambda_{2} z(x, t) \equiv\left\{\varepsilon a(x, t) \delta_{\bar{x} \widehat{x}}+b(x, t) \delta_{\widetilde{x}}\right\} z(x, t), \quad(x, t) \in G_{h} \cap G^{(1)},
\end{aligned}
$$

$\delta_{\widetilde{x}}$ is the first order central difference derivative;

$$
\begin{gathered}
\Lambda_{2} z(x, t) \equiv\left\{\begin{array}{c}
\left\{\varepsilon a(x, t) \delta_{\bar{x} \widehat{x}}+b(x, t) \delta_{\widetilde{x}}\right\} z(x, t), \text { if } \varepsilon N \geq M_{0} \\
\left\{\varepsilon a(x, t) \delta_{\bar{x} \widehat{x}}+b(x, t) \delta_{x}\right\} z(x, t), \text { if } \varepsilon N<M_{0}
\end{array}\right\}, \text { in } G_{h} \cap G^{(2)}, \\
\Lambda_{1} z(x, t) \equiv\left\{-p(x, t) \delta_{\bar{t}}-c(x, t)\right\} z(x, t), \quad(x, t) \in G_{h}, \\
\bar{G}^{(k)}=\bar{G}_{(3.4)}^{(k)}(\sigma), \sigma=\sigma_{(4.1)}, M_{0} \text { is any number satisfying the condition } \\
M_{0} \geq 2 d \max _{\bar{G}}\left(a^{-1}(x, t) b(x, t)\right) .
\end{gathered}
$$

Scheme (4.2), (4.1) is monotone $\varepsilon$-uniformly. For solutions of scheme (4.2), (4.1), using a priori estimates (3.3) and the maximum principle, we obtain the estimate for $(x, t) \in \bar{G}_{h}$ :

$$
|u(x, t)-z(x, t)| \leq\left\{\begin{array}{ll}
M\left[N^{-1}+N_{0}^{-1}\right] & \text { for } \varepsilon N<M_{0} \\
M\left[N^{-2}\left(\varepsilon+\ln ^{-1} N\right)^{-2}+N_{0}^{-1}\right] & \text { for } \varepsilon N \geq M_{0}
\end{array}\right\},
$$

where $M_{0}=M_{0(4.2)}$. We have also the $\varepsilon$-uniform estimate

$$
|u(x, t)-z(x, t)| \leq M\left[N^{-1}+N_{0}^{-1}\right], \quad(x, t) \in \bar{G}_{h} .
$$

Thus, scheme (4.2), (4.1) converges $\varepsilon$-uniformly with first-order accuracy.

Remark 2. The use of the first central difference derivative in $x$ for not too small values of the parameter $\varepsilon$, precisely, for

$$
\varepsilon N \geq M_{0(4.2)},
$$

allowed us to obtain the order of the convergence rate in $x$ close to two. According to (4.3),

$$
|u(x, t)-z(x, t)| \leq M\left[N^{-2}\left(\varepsilon+\ln ^{-1} N\right)^{-2}+N_{0}^{-1}\right], \quad(x, t) \in \bar{G}_{h},
$$

this estimate is unimprovable with respect to $N, N_{0}, \varepsilon$ (i.e. the estimate can not be improved with respect any one of the variables $N, N_{0}$ or $\varepsilon$ without loss of accuracy with respect to other variables). 
We now give a difference scheme of improved convergence rate with respect to $t$ under condition (4.4). For this we apply an approach based on the defect correction technique (see, e.g., [8]); we use scheme (4.2), (4.1) as the base scheme.

We approximate problem (2.2), (2.1) by the difference scheme

$$
\left\{\begin{array}{l}
\Lambda_{(4.2)}^{(2)} z^{(2)}(x, t)=f(x, t)+\psi^{0}(x, t), \quad(x, t) \in G_{h}, \\
z^{(2)}(x, t)=\varphi(x, t), \quad(x, t) \in S_{h} .
\end{array}\right.
$$

Here $\Lambda^{(2)}=\Lambda_{(4.2)}^{(2)}, \bar{G}_{h}=\bar{G}_{h(4.1)}, \psi^{0}(x, t)$ is the correcting term

$$
\psi^{0}(x, t)=\psi^{0}\left(x, t ; z^{(1)}(\cdot)\right) \equiv \frac{1}{2} \tau p(x, t)\left\{\begin{array}{l}
\delta_{2 \bar{t}} z^{(1)}(x, t), \quad t \geq 2 \tau \\
\frac{\partial^{2}}{\partial t^{2}} u(x, 0), \quad t=\tau
\end{array}\right\},
$$

$z^{(1)}(x, t),(x, t) \in \bar{G}_{h}$, is the solution of the discrete problem (4.2), (4.1), $\delta_{2 \bar{t}} z(x, t)$ is the backward second-order difference derivative

$$
\delta_{2 \bar{t}} z(x, t)=\left(\delta_{\bar{t}} z(x, t)-\delta_{\bar{t}} z(x, t-\tau)\right) / \tau, \quad(x, t) \in \bar{G}_{h}, \quad t \geq 2 \tau,
$$

$\tau$ is the step size of the mesh $\bar{\omega}_{0}$ in (4.1), $\tau=T N_{0}^{-1}$; the derivative $\frac{\partial^{2}}{\partial t^{2}} u(x, 0)$, $x \in \bar{D}$, can be found by virtue of the differential equation in (2.2). We call the function $z^{(2)}(x, t),(x, t) \in \bar{G}_{h}$, the solution of the difference scheme (4.5), (4.2), (4.1), (4.4). For simplicity, we assume that the coefficients $a(x, t), b(x, t)$ satisfy the condition

$$
a(x, t)=g(x) b(x, t), \quad(x, t) \in \bar{G}
$$

(for example, $a(x, t), b(x, t)$ do not depend on $t$ ), and the initial condition is homogeneous:

$$
\varphi(x, t)=0, \quad(x, t) \in S_{0} .
$$

Note that, under condition (4.7), the derivative $\frac{\partial^{2}}{\partial t^{2}} u(x, 0)$ required to compute the function $\psi_{(4.5 \mathrm{~b})}^{(0)}(x, t)$ for $t=\tau$ is determined by

$$
\begin{aligned}
\frac{\partial^{2}}{\partial t^{2}} u(x, 0)= & -p^{-1}(x, t)\left\{\varepsilon a(x, t) \frac{\partial^{2}}{\partial x^{2}}+b(x, t) \frac{\partial}{\partial x}-c(x, t)-\frac{\partial}{\partial t} p(x, t)\right\} \\
& \times\left\{p^{-1}(x, t) f(x, t)\right\}-p^{-1}(x, t) \frac{\partial}{\partial t} f(x, t), \quad(x, t) \in S_{0} .
\end{aligned}
$$

In the case of conditions (4.6), (4.7), the following estimate holds for the solution of scheme (4.5), (4.2), (4.1), (4.4) (see its proof for a linear problem, e.g., in [7]):

$$
\left|u(x, t)-z^{(2)}(x, t)\right| \leq M\left[N^{-2}\left(\varepsilon+\ln ^{-1} N\right)^{-2}+N_{0}^{-2}\right], \quad(x, t) \in \bar{G}_{h},
$$

this estimate is unimprovable with respect to $N, N_{0}, \varepsilon$. We have also the $\varepsilon$-uniform estimate

$$
\left|u(x, t)-z^{(2)}(x, t)\right| \leq M\left(N^{-2} \ln ^{2} N+N_{0}^{-2}\right), \quad(x, t) \in \bar{G}_{h} .
$$

Thus, for not too small values of the parameter $\varepsilon$ (for $\varepsilon N \geq M_{0(4.2)}$ ) the difference scheme (4.5), (4.2), (4.1), (4.4) converges $\varepsilon$-uniformly with the order of the convergence rate close to two in $x$ and equal to two in $t$. 
Theorem 4. Let conditions (4.6), (4.7), and also the estimates from Theorem 2 for $K=6$ be fulfilled. Then the difference scheme (4.5), (4.2), (4.1), (4.4) converges $\varepsilon$ uniformly. The numerical solutions under condition (4.4) satisfy estimates (4.8) and (4.9), which are unimprovable with respect to $N, N_{0}, \varepsilon$ and $N, N_{0}$, respectively.

\subsection{Schemes based on asymptotic constructs}

For small values of the parameter $\varepsilon$, we construct a higher-order accurate scheme by approximating problems (3.5a)-(3.5c).

The grid $\bar{G}_{h(4.1)}$ is decomposed into the sum of grid sets

$$
\bar{G}_{h}=\bar{G}_{h}^{(1)} \cup \bar{G}_{h}^{(2)}, \bar{G}_{h}^{(k)}=\bar{G}^{(k)} \cap \bar{G}_{h}, \quad \Gamma_{h}^{(k)}=\Gamma^{(k)} \cap \bar{G}_{h}, \quad k=1,2,
$$

where

$$
\bar{G}^{(k)}=\bar{G}_{(3.4)}^{(k)}=\bar{G}_{(3.4)}^{(k)}(\sigma), \quad \sigma=\sigma_{(4.1)} .
$$

On the grid $\bar{G}_{h}^{(2)}$, we approximate problem (3.5a), (3.5b) by the difference scheme

$$
\begin{gathered}
\left\{\begin{array}{l}
\Lambda^{(1)} z_{0}^{2}(x, t)=f(x, t), \quad(x, t) \in G_{h}^{(2)} \\
z_{0}^{2}(x, t)=\varphi(x, t), \quad(x, t) \in S_{h}^{(2)}
\end{array}\right. \\
\left\{\begin{array}{l}
\Lambda^{(1)} z_{1}^{2}(x, t)=-a(x, t) \delta_{x \bar{x}} z_{0}^{2}(x, t)+\psi^{1}(x, t)+\psi^{0}(x, t),(x, t) \in G_{h}^{(2)}, \\
z_{1}^{2}(x, t)=0, \quad(x, t) \in S_{h}^{(2)} .
\end{array}\right.
\end{gathered}
$$

Here

$$
\begin{aligned}
& \Lambda^{(1)} z(x, t) \equiv\left\{b(x, t) \delta_{x}-c(x, t)-p(x, t) \delta_{\bar{t}}\right\} z(x, t), \quad(x, t) \in G_{h}^{(2)}, \\
& \psi^{1}(x, t) \equiv 2^{-1} \varepsilon^{-1} h^{i} b(x, t)\left\{\begin{array}{l}
\delta_{x \bar{x}} z_{0}^{2}(x, t), x>\sigma \\
\delta_{x \bar{x}} z_{0}^{2}(\widehat{x}, t), x=\sigma
\end{array}\right\}, \quad h^{i}=x^{i+1}-x^{i}, \\
& \psi^{0}(x, t)=\varepsilon^{-1} \psi_{(4.5)}^{0}\left(x, t ; z_{0}^{(2)}(\cdot)\right), \\
& \delta_{x \bar{x}} z_{0}^{2}(\widehat{x}, t)=\delta_{x \bar{x}} z_{0}^{2}\left(x^{i+1}, t\right), \quad x=x^{i} .
\end{aligned}
$$

The function

$$
z^{2}(x, t)=z_{0}^{2}(x, t)+\varepsilon z_{1}^{2}(x, t), \quad(x, t) \in \bar{G}_{h}^{(2)},
$$

is called the solution of problem (4.11a), (4.11b). The operator $\Lambda^{(1)}$ is monotone.

For the function $z^{2}(x, t),(x, t) \in \bar{G}_{h}^{(2)}$, we obtain the estimate

$$
\left|u^{2}(x, t)-z^{2}(x, t)\right| \leq M\left(\varepsilon^{2}+N^{-2}+N_{0}^{-2}\right), \quad(x, t) \in \bar{G}_{h}^{(2)} .
$$

Note that the function $z_{0(4.11 \mathrm{a})}^{2}(x, t),(x, t) \in \bar{G}_{h}^{(2)}$, approximates the function $u_{0(3.5 \mathrm{a})}^{2}(x, t)$ only with the first-order accuracy. The use of the additional correcting terms 


$$
\frac{1}{2}\left(h^{i} b(x, t) \delta_{x \bar{x}}+\tau p(x, t) \delta_{2 \bar{t}}\right) z_{0}^{2}(x, t)
$$

in the equation from (4.11b) to compute the function $z_{1}^{2}(x, t)$ allows us to improve the approximation of the function $u_{(3.5 \mathrm{a} ; 3.5 \mathrm{~b})}^{2}(x, t)$ and the accuracy of the numerical solution of problem (2.2), (2.1) on $\bar{G}^{(2)}$ (see estimate (4.12) on $\bar{G}_{h}^{(2)}$ ).

On the grid $\bar{G}_{h(4.10)}^{(1)}$, problem (3.5c) is approximated by the difference scheme

$$
\begin{gathered}
\left\{\begin{array}{c}
\Lambda^{1} z^{1}(x, t) \equiv\left\{\varepsilon a(x, t) \delta_{x \bar{x}}+b(x, t) \delta_{\widetilde{x}}-c(x, t)-p(x, t) \delta_{\bar{t}}\right\} z^{1}(x, t) \\
=f(x, t), \quad(x, t) \in G_{h}^{(1)},
\end{array}\right. \\
z^{1}(x, t)= \begin{cases}\varphi(x, t), & (x, t) \in S_{h}^{(1)} \bigcap S, \\
z^{2}(x, t), & (x, t) \in S_{h}^{(1)} \backslash S .\end{cases}
\end{gathered}
$$

The operator $\Lambda^{1}$ is monotone on the grid set $G_{h}^{(1)}$. The proximity of the solutions of the boundary value problem (3.5c) on the set $\bar{G}_{h}^{(1)}$ and of its numerical approximation (4.13) follows from the a priori estimates for the solution of the differential problem and from the proximity of the functions $u^{2}(x, t)$ and $z^{2}(x, t)$ on the set $S_{h}^{(1)} \cap \bar{G}^{(2)}$. For the function $z^{1}(x, t),(x, t) \in \bar{G}_{h}^{(1)}$, we obtain the estimate

$$
\left|u^{1}(x, t)-z^{1}(x, t)\right| \leq M\left(\varepsilon^{2}+\varepsilon \frac{\ln N}{N_{0}}+N^{-2}\left(\varepsilon+\ln ^{-1} N\right)^{-2}+N_{0}^{-2}\right) .
$$

On the grid $\bar{G}_{h(4.1)}$, we define the function $z(x, t)$ by the relation

$$
z(x, t)=\left\{z^{k}(x, t), \quad(x, t) \in \bar{G}_{h}^{(k)}, \quad k=1,2\right\}, \quad(x, t) \in \bar{G}_{h},
$$

where $z^{k}(x, t),(x, t) \in \bar{G}_{h}^{(k)}$, are the solutions of problems (4.11a; 4.11b) and (4.13). The function $z_{(4.15)}(x, t),(x, t) \in \bar{G}_{h}$, is called the solution of the difference scheme (4.11), (4.1).

For the solution of scheme (4.11), (4.1), by virtue of bound (4.14), we have the estimate for $(x, t) \in \bar{G}_{h}$ :

$$
|u(x, t)-z(x, t)| \leq M\left(\varepsilon^{2}+\varepsilon \frac{\ln N}{N_{0}}+N^{-2}\left(\varepsilon+\ln ^{-1} N\right)^{-2}+N_{0}^{-2}\right) .
$$

Remark 3. For sufficiently small values of the parameter $\varepsilon$ (for $\varepsilon \leq M N^{-1}$ ) scheme (4.11), (4.1) converges $\varepsilon$-uniformly at the rate $\mathcal{O}\left(N^{-2} \ln ^{2} N+N_{0}^{-2}\right)$ :

$$
|u(x, t)-z(x, t)| \leq M\left(N^{-2} \ln ^{2} N+N_{0}^{-2}\right), \quad(x, t) \in \bar{G}_{h},
$$

this estimate is unimprovable (with respect to $N, N_{0}$ for $\varepsilon \leq M N^{-1}$ ).

The scheme of improved $\varepsilon$-uniform convergence order for $\varepsilon \in(0,1]$ is constructed on the basis of schemes (4.5), (4.2), (4.1) and (4.11), (4.1). For $\varepsilon \geq \varepsilon_{0}$, where

$$
\varepsilon_{0}=\varepsilon_{0}(N)=M_{(4.2)}^{0} N^{-1},
$$


we use scheme (4.5), (4.2), (4.1), (4.4), while for $\varepsilon<\varepsilon_{0}$ we use scheme (4.11), (4.1). The function $z(x, t),(x, t) \in \bar{G}_{h}$, constructed in this way is called the solution of the difference scheme (4.5), (4.2), (4.11), (4.18), (4.1).

From estimates (4.8), (4.9) and (4.17) it follows that the solution of scheme (4.5), (4.2), (4.11), (4.18), (4.1) satisfies the estimate

$$
|u(x, t)-z(x, t)| \leq M\left\{\begin{array}{ll}
N^{-2} \ln ^{2} N+N_{0}^{-2}, & \varepsilon<\varepsilon_{0} \\
N^{-2}\left(\varepsilon+\ln ^{-1} N\right)^{-2}+N_{0}^{-2}, & \varepsilon \geq \varepsilon_{0}
\end{array}\right\}, \quad(x, t) \in \bar{G}_{h},
$$

and also the $\varepsilon$-uniform estimate

$$
|u(x, t)-z(x, t)| \leq M\left[N^{-2} \ln ^{2} N+N_{0}^{-2}\right], \quad(x, t) \in \bar{G}_{h} .
$$

Estimates (4.19a) and (4.19b) are unimprovable with respect to $N, N_{0}, \varepsilon$ and $N, N_{0}$, respectively. Scheme (4.5), (4.2), (4.11), (4.18), (4.1) converges $\varepsilon$-uniformly with the second order in $x$ up to a logarithmic factor and with the second order in $t$.

Theorem 5. Let the data of the boundary value problem (2.2), (2.1) and its solutions satisfy the hypotheses of Theorem 4 . Then the solutions of the difference scheme (4.5), (4.2), (4.11), (4.18), (4.1) as $N, N_{0} \rightarrow \infty$ converges to the solution of problem (2.2), (2.1) E-uniformly at the rate $\mathcal{O}\left(N^{-2} \ln ^{2} N+N_{0}^{-2}\right)$. The numerical solutions satisfy the unimprovable estimates (4.19).

Remark 4. For fixed values of the parameter $\varepsilon$, scheme (4.5), (4.2), (4.11), (4.18), (4.1) converges with the second-order accuracy.

\section{Statement of the Problem with Piecewise Smooth Initial Data. Aim of Study}

In the domain $\bar{G}$ with boundary $S$, where

$$
\bar{G}=G \cup S, \quad G=D \times(0, T], \quad D=\{x: x \in(-d, d)\},
$$

we consider the Dirichlet problem for the singularly perturbed parabolic equation with constant coefficients and piecewise-smooth initial data

$$
\left\{\begin{array}{l}
L_{(5.2)} u(x, t)=f(x, t), \quad(x, t) \in G, \\
u(x, t)=\varphi(x, t), \quad(x, t) \in S .
\end{array}\right.
$$

Here

$$
L_{(5.2)} \equiv \varepsilon a \frac{\partial^{2}}{\partial x^{2}}+b \frac{\partial}{\partial x}-c-p \frac{\partial}{\partial t},
$$

$a, b, p>0, c \geq 0$, the right-hand side $f(x, t)$ is sufficiently smooth on $\bar{G}$. The boundary function $\varphi(x, t)$ is continuous on $S$, sufficiently smooth on the set $S^{L}$, and piecewise smooth on the set $S_{0}$. For $t=0$ the first $x$-derivative of the function $\varphi(x, t)$ has a discontinuity of the first kind on the set $S^{(*)}$. Here 


$$
S^{*}=S \backslash S^{(*)}, S^{(*)}=\{(x, t): x=t=0\}, \quad S=S^{L} \bigcup S_{0},
$$

$S^{L}$ and $S_{0}=\bar{S}_{0}$ are the lateral and lower parts of the boundary $S$,

$$
S^{L}=S^{l} \bigcup S^{r}, \quad S_{0}^{*}=S_{0} \backslash S^{(*)}
$$

where $S^{l}$ and $S^{r}$ are the left and right parts of the lateral boundary $S^{L}$.

By a solution of problem (2.2), we mean a function $u \in C(\bar{G}) \cap C^{2,1}(G)$ that satisfies the differential equation on $G$ and the boundary condition on $S$. For simplicity, we assume that compatibility conditions ensuring local smoothness of the solution for fixed $\varepsilon$ [11] are fulfilled on the set $S_{*}=S_{0} \cap \bar{S}^{L}$. Suppose that on the set $\bar{G}^{\delta}$, i.e., the $\delta$-neighbourhood of the set $S_{*}$, the inclusion

$$
u \in C^{l+\alpha,(l+\alpha) / 2}\left(\bar{G}^{\delta}\right), \quad l \geq 2, \quad \alpha \in(0,1)
$$

is satisfied. The derivative $\frac{\partial}{\partial x} u(x, t)$ is continuous on $\bar{G}^{*}$, where $\bar{G}^{*}=\bar{G} \backslash S^{(*)}$; for fixed $\varepsilon$, it is bounded on $\bar{G}^{*}$ and discontinuous on $S^{(*)}$.

We are interested in a numerical approximation to the solution $u(x, t),(x, t) \in$ $\bar{G}$. Let us specify the behaviour of the solution. Let

$$
S^{\gamma}=\{(x, t): x=\gamma(t), \quad(x, t) \in \bar{G}\},
$$

and let $x=\gamma(t), t \geq 0$, be the characteristic of the reduced equation passing through the point $(0,0)$. As $\varepsilon \rightarrow 0$, in the neighbourhood of the sets $S^{l}$ and $S^{\gamma}$ there appear boundary and transient layers with typical scales $\varepsilon$ and $\varepsilon^{1 / 2}$, respectively, unlike the boundary layer, the transient layer is weak (the first $x$-derivative of the transient-layer function is bounded $\varepsilon$-uniformly; see estimates (6.4) in Section 6). For simplicity, we assume that the characteristic $S^{\gamma}$ does not meet the boundary $S^{l}$, that is, the transient and boundary layers do not interact.

The classical finite difference scheme for problem (5.2), (5.1) in the case of piecewise uniform meshes condensing in the layers converges $\varepsilon$-uniformly at the rate $\mathcal{O}\left(N^{-1 / 2}+N_{0}^{-1 / 2}\right.$ ) (see estimate (7.6) for solutions of scheme (7.2), (7.5) in Section 7). This rate of convergence is essentially lower than that for problems with sufficiently smooth data.

Our aim is to construct a numerical method that converges $\varepsilon$-uniformly at the rate $\mathcal{O}\left(N^{-1} \ln N+N_{0}^{-1}\right)$ for the problem (5.2), (5.1).

Note that under condition (7.9) below, when the main term of the transient layer (the function $W_{1}(x, t)$ in representation (6.3)) vanishes (see (7.8)), the classical difference scheme on a piecewise uniform mesh converges $\varepsilon$-uniformly at the rate $\mathcal{O}\left(N^{-1} \ln N+N_{0}^{-1}\right)$. Thus, to construct a scheme with improved convergence it seems appropriate to apply the method of the additive splitting of singularities when the component $W_{1}(x, t)$ generated by the discontinuity in the derivative $\frac{\partial}{\partial x} \varphi(x, 0)$ is separated. The remaining part of the solution (the function $\left.w(x, t)=u(x, t)-W_{1}(x, t)\right)$, which is a solution of the boundary value problem with an initial function having continuous first $x$-derivative, can be found using schemes on piecewise uniform meshes. Such an approach is applied here to construct an improved scheme for problem (5.2), (5.1). 


\section{A Priori Estimates for Problem (5.2), (5.1)}

To derive estimates for the solution of problem (5.2) and its derivatives, we apply the technique from $[16,17,18]$. We assume that the functions $f(x, t)$ and $\varphi(x, t)$ are sufficiently smooth on the sets $\bar{G}$ and $\bar{S}^{L}, \bar{S}_{0+}, \bar{S}_{0-}$, respectively, and also the solution of the problem satisfies condition (5.3), where $l \geq K-2, K \geq 4$.

The set $\bar{G}$ is decomposed into the sum of overlapping sets

$$
\bar{G}=\bigcup_{j} \bar{G}^{j}, \quad j=1,2,3,
$$

where

$$
\begin{aligned}
& G^{1}=G^{1}\left(m^{1}\right)=\left\{(x, t):|x-\gamma(t)|<m^{1}, t \in(0, T]\right\}, \\
& G^{2}=G^{2}\left(m^{2}\right)=\left\{(x, t): x \in\left(-d,-d+m^{2}\right), t \in(0, T]\right\}, \\
& G^{3}=G^{3}\left(m^{3}\right)=G \backslash\left\{G^{1}\left(m^{3}\right) \cup G^{2}\left(m^{3}\right)\right\}, \quad m^{3}<m^{1}, m^{2},
\end{aligned}
$$

$G^{1}$ and $G^{2}$ are the neighbourhoods of the transient and boundary layers, respectively; let $\bar{G}^{1} \cap \bar{G}^{2}=\emptyset$. We denote the solution of problem (5.2), (5.1) considered on the set $\bar{G}^{j}$ by $u^{j}(x, t), j=1,2,3$. Using the results from $[16,17,18]$ we find the estimate

$$
\left|\frac{\partial^{k+k_{0}}}{\partial x^{k} \partial t^{k_{0}}} u^{3}(x, t)\right| \leq M, \quad(x, t) \in \bar{G}^{3}, \quad k+2 k_{0} \leq K .
$$

The value of $K$ is defined by the problem data; $K \geq 4$.

Let us discuss a behaviour of the solution $u^{1}(x, t)$ on the set $\bar{G}^{1}$. We represent the function $u^{1}(x, t),(x, t) \in \bar{G}^{1}$, in the form of the sum of functions

$$
u^{1}(x, t)=U^{1}(x, t)+W^{1}(x, t), \quad(x, t) \in \bar{G}^{1},
$$

where $U^{1}(x, t)$ and $W^{1}(x, t)$ are the regular and singular parts of the solutions,

$$
\begin{aligned}
& U^{1}(x, t)=U^{1}(x, t ; i)=U(x, t)+\sum_{k=i+1}^{K-1} W_{k}(x, t), \quad(x, t) \in \bar{G}^{1} ; \\
& W^{1}(x, t)=W^{1}(x, t ; i)=\sum_{k=1}^{i} W_{k}(x, t), \quad(x, t) \in \bar{G},
\end{aligned}
$$

and $i$ takes one of the values, either 1 or 2 . Here $U(x, t),(x, t) \in \bar{G}^{1}$, is the component of the solution to the inhomogeneous equation from (5.2) having $\varepsilon$-uniformly bounded derivatives in $x$ and $t$ up to the orders $K$ and $2^{-1} K$ respectively. The functions $W_{k}(x, t),(x, t) \in \mathbb{R} \times[0, T]$, are solutions of the Cauchy problems

$$
\begin{aligned}
& L_{(5.2)} W_{k}(x, t)=0, \quad(x, t) \in \mathbb{R} \times(0, T], \quad k=1, \ldots, K-1, \\
& W_{k}(x, 0)=2^{-1}(k !)^{-1}\left[\frac{\partial^{k}}{\partial x^{k}} \varphi(x, 0)\right]|x| x^{k-1}, \quad x \in \mathbb{R} .
\end{aligned}
$$


Here

$$
\left[\frac{\partial^{k}}{\partial x^{k}} \varphi(x, 0)\right]=\frac{\partial^{k}}{\partial x^{k}} \varphi(+0,0)-\frac{\partial^{k}}{\partial x^{k}} \varphi(-0,0), \quad x=0, \quad k \geq 1
$$

is the jump of the derivative $\frac{\partial^{k}}{\partial x^{k}} \varphi(x, 0)$ when passing through the point $x=0$. For example, the function $W_{1}(x, t)$ is defined by

$$
\begin{aligned}
& W_{1}(x, t)= \\
& =\frac{1}{2}\left[\frac{\partial}{\partial x} \varphi(0,0)\right]\left\{(x-\gamma(t)) v\left(2^{-1} \varepsilon^{-1 / 2} a^{-1 / 2} p^{1 / 2}(x-\gamma(t)) t^{-1 / 2}\right)\right. \\
& \left.+2 \pi^{-1 / 2} \varepsilon^{1 / 2} a^{1 / 2} p^{-1 / 2} t^{1 / 2} \exp \left(-4^{-1} \varepsilon^{-1} a^{-1} p(x-\gamma(t))^{2} t^{-1}\right)\right\} \exp (-\alpha t), \\
& v(\xi)=\operatorname{erf}(\xi)=2 \pi^{-1 / 2} \int_{0}^{\xi} \exp \left(-\alpha^{2}\right) d \alpha, \quad \xi \in \mathbb{R} ; \gamma(t)=-b p^{-1} t, \alpha=c p^{-1} .
\end{aligned}
$$

For the components in representation (6.3), taking into account the explicit form of the functions $W_{k}(x, t), k=1, \ldots, K-1$, we find the estimates

$$
\begin{gathered}
\left|\frac{\partial^{k+k_{0}}}{\partial x^{k} \partial t^{k_{0}}} U^{1}(x, t)\right| \leq M\left[1+\varepsilon^{\left(i+1-k-k_{0}\right) / 2} \rho^{i+1-k-k_{0}}\right. \\
\left.+\varepsilon^{(i+1-k) / 2} \rho^{i+1-k-2 k_{0}}\right], \quad(x, t) \in \bar{G}^{1}, \\
\left|\frac{\partial^{k+k_{0}}}{\partial x^{k} \partial t^{k_{0}}} W^{1}(x, t)\right| \leq M\left[1+\varepsilon^{\left(1-k-k_{0}\right) / 2} \rho^{1-k-k_{0}}+\varepsilon^{(1-k) / 2} \rho^{1-k-2 k_{0}}\right], \\
(x, t) \in \bar{G} ; \quad k+2 k_{0} \leq K, \quad i=1,2,
\end{gathered}
$$

where

$$
\rho=\rho(x, t ; \varepsilon)=\varepsilon^{-1 / 2}|x-\gamma(t)|+t^{1 / 2},
$$

$m$ is an arbitrary constant.

We now consider the solution $u^{2}(x, t)$ of problem $(5.2),(5.1)$ on the set $\bar{G}^{2}$. The solution can be represented as the sum of functions

$$
u^{2}(x, t)=U^{2}(x, t)+V^{2}(x, t), \quad(x, t) \in \bar{G}^{2},
$$

where $U^{2}(x, t)$ and $V^{2}(x, t)$ are the regular and singular parts of the solution. The function $U^{2}(x, t)$ is the restriction of the function $U^{e}(x, t),(x, t) \in \bar{G}^{2 e}$, to the set $\bar{G}^{2}$. Here $U^{e}(x, t)$ is a solution of the problem

$$
\left\{\begin{array}{l}
L_{(5.2)} U^{e}(x, t)=f^{e}(x, t), \quad(x, t) \in G^{2 e} \\
U^{e}(x, t)=\varphi^{e}(x, t), \quad(x, t) \in S^{2 e}
\end{array}\right.
$$

The domain $G^{2 e}$ is an extension of the domain $G^{2}$ beyond the boundary $S^{l}$. The right-hand side $f^{e}(x, t)$ is a smooth continuation of the function $f(x, t)$. The function 
$\varphi^{e}(x, t)$ is smooth on each piecewise-smooth part of the set $S^{2 e}$, and it coincides with the functions $\varphi(x, t)$ and $u^{1}(x, t)$ on the sets $S^{2} \cap S_{0}$ and $S^{2} \cap G^{1}$, respectively. The function $V^{2}(x, t)$ is the solution of the problem

$$
\begin{cases}L_{(5.2)} V^{2}(x, t)=0, & (x, t) \in G^{2}, \\ V^{2}(x, t)=\varphi(x, t)-U^{2}(x, t), & (x, t) \in S^{l}, \\ V^{2}(x, t)=0, & (x, t) \in S^{2} \backslash S^{l} .\end{cases}
$$

For the functions $U^{2}(x, t)$ and $V^{2}(x, t)$, the following estimates are valid:

$$
\begin{aligned}
& \left|\frac{\partial^{k+k_{0}}}{\partial x^{k} \partial t^{k_{0}}} U^{2}(x, t)\right| \leq M, \quad(x, t) \in \bar{G}^{2}, \quad k+2 k_{0} \leq K, \\
& \left|\frac{\partial^{k+k_{0}}}{\partial x^{k} \partial t^{k_{0}}} V^{2}(x, t)\right| \leq M \varepsilon^{-k} \exp \left(-m \varepsilon^{-1} r\left((x, t), \bar{S}^{l}\right)\right),
\end{aligned}
$$

where $r\left((x, t), \bar{S}^{l}\right)$ is the distance from the point $(x, t)$ to the set $\bar{S}^{l}, m$ is any constant from the interval $\left(0, m_{0}\right), m_{0}=a^{-1} b$.

Theorem 6. Let the data of the boundary value problem (5.2), (5.1) satisfy the condition $f \in C^{l_{1}, l_{1} / 2}(\bar{G}), \varphi \in C^{l_{1}}\left(\bar{S}_{0}^{-}\right) \cap C^{l_{1}}\left(\bar{S}_{0}^{+}\right) \cap C^{l_{1} / 2}\left(\bar{S}^{L}\right) \cap C(S)$, $l_{1}=l+\alpha$, and let the solution $u$ of the problem satisfy condition (5.3), where $l=K, \alpha \in(0,1)$. Then the solution $u$ and its components in representations (6.3), (6.5) satisfy estimates (6.2), (6.4), (6.6).

\section{Classical Finite Difference Approximations on Uniform and Piecewise-Uniform Meshes. Problem (5.2), (5.1)}

We construct a difference scheme that allows us to approximate the solution of problem (5.2), (5.1) $\varepsilon$-uniformly.

First we consider a difference scheme based on classical approximations. On the set $\bar{G}_{(5.1)}$, we introduce the rectangular grid

$$
\bar{G}_{h}=\bar{D}_{h} \times \bar{\omega}_{0}=\bar{\omega} \times \bar{\omega}_{0},
$$

where $\bar{\omega}$ and $\bar{\omega}_{0}$ are meshes on the segments $[-d, d]$ and $[0, T]$, respectively, the mesh $\bar{\omega}$ has an arbitrary distribution of its nodes satisfying only the condition $h \leq M N^{-1}$, where $h=\max _{i} h^{i}, h^{i}=x^{i+1}-x^{i}, x^{i}, x^{i+1} \in \bar{\omega}$, the mesh $\bar{\omega}_{0}$ is uniform with step-size $\tau=T N_{0}^{-1}$. Here $N+1$ and $N_{0}+1$ are the numbers of nodes in the meshes $\bar{\omega}$ and $\bar{\omega}_{0}$, respectively.

We approximate problem (5.2) by the difference scheme [14]

$$
\left\{\begin{array}{l}
\Lambda_{(7.2)} z(x, t)=f(x, t), \quad(x, t) \in G_{h}, \\
z(x, t)=\varphi(x, t), \quad(x, t) \in S_{h},
\end{array}\right.
$$

where 


$$
\Lambda_{(7.2)} \equiv \varepsilon a \delta_{\bar{x} \widehat{x}}+b \delta_{x}-c-p \delta_{\bar{t}} .
$$

Consider the difference scheme (7.2) on the uniform mesh

$$
\bar{G}_{h}=\bar{G}_{h}^{u}=\bar{\omega} \times \bar{\omega}_{0} .
$$

Using a priori estimates (6.2), (6.4), (6.6) for the solutions of problem (5.2), we find the estimate

$$
|u(x, t)-z(x, t)| \leq M\left(\left(\varepsilon+N^{-1}\right)^{-1} N^{-1}+N^{-1 / 2}+N_{0}^{-1 / 2}\right), \quad(x, t) \in \bar{G}_{h} .
$$

Thus, scheme (7.2), (7.3) converges for fixed values of the parameter $\varepsilon$, at the rate $\mathcal{O}\left(N^{-1 / 2}+N_{0}^{-1 / 2}\right)$.

We now construct a difference scheme convergent $\varepsilon$-uniformly. On the set $\bar{G}$, we construct a mesh condensing in a neighbourhood of the boundary layer, similar to that constructed in $[2,12,16,17,18]$,

$$
\bar{G}_{h}=\bar{D}_{h} \times \bar{\omega}_{0}=\bar{\omega}^{*} \times \bar{\omega}_{0},
$$

where $\bar{\omega}_{0}=\bar{\omega}_{0(7.1)}, \bar{\omega}^{*}=\bar{\omega}^{*}(\sigma)$ is a piecewise uniform mesh on $[-d, d], \sigma$ is a mesh parameter depending on $\varepsilon$ and $N$. The value of $\sigma$ is chosen to satisfy the condition

$$
\sigma=\sigma(N, \varepsilon)=\min \left(\beta, 2 m^{-1} \varepsilon \ln N\right),
$$

where $\beta$ is an arbitrary number in the half-open interval $(0, d], m=m_{(6.6)}$. The segment $[-d, d]$ is divided into two parts $[-d,-d+\sigma]$ and $[-d+\sigma, d]$; on each part the mesh step-size is constant and equal to $h^{(1)}=2 d \sigma \beta^{-1} N^{-1}$ on $[-d,-d+\sigma]$ and $h^{(2)}=2 d(2 d-\sigma)(2 d-\beta)^{-1} N^{-1}$ on $[-d+\sigma, d], \sigma \leq d$.

The difference scheme (7.2), (7.5) converges $\varepsilon$-uniformly with an error bound given by

$$
|u(x, t)-z(x, t)| \leq M\left(N^{-1 / 2}+N_{0}^{-1 / 2}\right), \quad(x, t) \in \bar{G}_{h} .
$$

Theorem 7. Let the solution of problem (5.2), (5.1) and its components in representations (6.3), (6.5) satisfy estimates (6.2), (6.4), (6.6) for $K=4$. Then the difference scheme (7.2), (7.5) (scheme (7.2), (7.3)) converges $\varepsilon$-uniformly (for fixed values of the parameter) at the rate $\mathcal{O}\left(N^{-1 / 2}+N_{0}^{-1 / 2}\right)$. The numerical solutions satisfy estimates (7.4), (7.6).

Remark 5. If the component $V(x, t)$ is absent in representation (6.5), i.e., if

$$
V(x, t)=0, \quad(x, t) \in \bar{G}^{2},
$$

we have the error estimate

$$
|u(x, t)-z(x, t)| \leq M\left(N^{-1 / 2}+N_{0}^{-1 / 2}\right), \quad(x, t) \in \bar{G}_{h} .
$$

Thus, under condition (7.7) scheme (7.2), (7.3) converges $\varepsilon$-uniformly and the convergence rate is $\mathcal{O}\left(N^{-1 / 2}+N_{0}^{-1 / 2}\right)$. Provided that 


$$
f(x, t)=0, \quad(x, t) \in \bar{G}, \quad \varphi(x, t)=0, \quad(x, t) \in S^{l},
$$

the boundary layer is absent, so condition (7.7) holds. However, the condition

$$
f(x, t)=0, \quad(x, t) \in \bar{G}^{2}, \quad \varphi(x, t)=0, \quad(x, t) \in S \cap \bar{G}^{2},
$$

is generally not sufficient for (7.7) to be valid.

Remark 6. On the mesh (7.5), the numerical solution approximates the singular component $V(x, t) \varepsilon$-uniformly with accuracy $\mathcal{O}\left(N^{-1} \ln N+N_{0}^{-1}\right)$.

Remark 7. Let the component $W_{1}(x, t)$ be absent in representation (6.3), i.e.,

$$
W_{1}(x, t)=0, \quad(x, t) \in \bar{G}^{1} ;
$$

in this case the derivative $(\partial / \partial x) \varphi(x, t)$ is continuous on $S_{0}$ and satisfy on $S^{(*)}$ the relation

$$
\left[\frac{\partial}{\partial x} \varphi(x, t)\right]=0, \quad(x, t) \in S^{(*)} .
$$

When using scheme (7.2), (7.5), we obtain the estimate

$$
|u(x, t)-z(x, t)| \leq M\left(N^{-1} \ln N+N_{0}^{-1}\right), \quad(x, t) \in \bar{G}_{h} .
$$

In the case of conditions (7.7) and (7.8), on the mesh (7.3) we have the estimate

$$
|u(x, t)-z(x, t)| \leq M\left(N^{-1}+N_{0}^{-1}\right), \quad(x, t) \in \bar{G}_{h} .
$$

\section{Improved Scheme of the Additive Splitting of Singularities for Problem (5.2), (5.1)}

To construct the improved difference scheme (in comparison to scheme (7.2), (7.5)) we apply the method of splitting singularities.

The solution of problem (5.2), (5.1) is decomposed into the sum of functions

$$
u(x, t)=u_{1}(x, t)+u_{2}(x, t), \quad(x, t) \in \bar{G},
$$

where

$$
u_{2}(x, t)=W^{1}(x, t)=W_{(6.3)}^{1}(x, t ; i), \quad(x, t) \in \bar{G}, \quad i=1 .
$$

The function $u_{1}(x, t)$ is a solution of the problem

$$
\left\{\begin{array}{l}
L_{(5.2)} u_{1}(x, t)=f(x, t), \quad(x, t) \in G, \\
u_{1}(x, t)=\varphi_{1}(x, t), \quad(x, t) \in S,
\end{array}\right.
$$

where

$$
\varphi_{1}(x, t)=\varphi(x, t)-W^{1}(x, t), \quad(x, t) \in S,
$$


the function $\varphi_{1}(x, t)$ and its first derivative in $x$ are continuous on $S_{0}$.

We approximate problem (8.1b) on mesh (7.1) by the difference scheme

$$
\begin{aligned}
\Lambda_{(7.2)} z_{1}(x, t) & =f(x, t), & & (x, t) \in G_{h}, \\
z_{1}(x, t) & =\varphi_{1}(x, t), & & (x, t) \in S_{h} .
\end{aligned}
$$

Using the function $z_{1}(x, t),(x, t) \in \bar{G}_{h}$, we construct the function $\bar{z}_{1}(x, t)$, $(x, t) \in \bar{G}$, which is the extension of $z_{1}(x, t),(x, t) \in \bar{G}_{h}$, onto $\bar{G}$; namely, $\bar{z}_{1}(x, t)$ is the bilinear interpolant on elementary rectangles generated by straight lines parallel to the coordinate axes and passing through the nodes of the mesh $\bar{G}_{h}$. Further, we construct the function

$$
u_{0}^{h}(x, t)=\bar{z}_{1}(x, t)+u_{2}(x, t), \quad(x, t) \in \bar{G},
$$

where $u_{2}(x, t)=u_{2(8.1 a)}(x, t)$. The function $u_{0}^{h}(x, t),(x, t) \in \bar{G}$, is called the solution of scheme (8.2), (7.1), which is the scheme based on the method of the additive splitting of singularities (namely, the main term of the transient layer).

Consider the difference scheme (8.2), (7.5). Taking estimates (6.4) into account, we have

$$
\left|u_{1}(x, t)-z_{1}(x, t)\right| \leq M\left[N^{-1} \ln N+N_{0}^{-1}\right], \quad(x, t) \in \bar{G}_{h} .
$$

For the function $u_{0}^{h}(x, t),(x, t) \in \bar{G}$, we obtain the estimate

$$
\left|u(x, t)-u_{0}^{h}(x, t)\right| \leq M\left[N^{-1} \ln N+N_{0}^{-1}\right], \quad(x, t) \in \bar{G} .
$$

Scheme (8.2), (7.5) converges $\varepsilon$-uniformly at the rate $\mathcal{O}\left(N^{-1} \ln N+N_{0}^{-1}\right)$.

Theorem 8. Let the hypothesis of Theorem 7 be fulfilled. Then the difference scheme (8.2), (7.5) converges $\varepsilon$-uniformly with the error bound (8.3).

The author is grateful to Pieter W. Hemker for interest discussions of high-order accurate numerical methods for singularly perturbed problems and the participants of the 10th International Conference MMA2005\&CMAM2 (Trakai, Lithuania, 2005) expressed a deep interest in results of the present paper.

\section{References}

[1] N.S. Bakhvalov. On the optimization of methods for boundary-value problems with boundary layers. Zh. Vychisl. Mat. Mat. Fiz., 9(4), 841 - 859, 1969. (in Russian)

[2] P.A. Farrell, A.F. Hegarty, J.J.H. Miller, E. O'Riordan and G.I. Shishkin. Robust computational techniques for boundary layers. Chapman and Hall/CRC Press, Boca Raton, 2000.

[3] P.W. Hemker, G.I. Shishkin and L.P. Shishkina. The use of defect correction for the solution of parabolic singular perturbation problems. ZAMM-Z. Angew. Math. Mech., 77(1), 59 - 74, 1997.

[4] P.W. Hemker, G.I. Shishkin and L.P. Shishkina. $E$-uniform schemes with high-order time-accuracy for parabolic singular perturbation problems. IMA J. Numer. Anal., 20(1), $99-121,2000$. 
[5] P.W. Hemker, G.I. Shishkin and L.P. Shishkina. High-order time-accurate parallel schemes for parabolic singularly perturbed problems with convection. Computing, 66 (2), $139-161,2001$.

[6] P.W. Hemker, G.I. Shishkin and L.P. Shishkina. High-order time-accurate schemes for parabolic singular perturbation convection-diffusion problems with robin boundary conditions. Comput. Methods Appl. Math., 2(1), 3-25, 2002.

[7] P.W. Hemker, G.I. Shishkin and L.P. Shishkina. High-order time-accurate schemes for parabolic singular perturbation problems with convection. Russian J. Numer. Anal. Math. Modelling, 17(1), 1-24, 2002.

[8] P.W. Hemker, G.I. Shishkin and L.P. Shishkina. Novel defect correction high-order, in space and time, accurate schemes for parabolic singularly perturbed convection-diffusion problems. Comput. Methods Appl. Math., 3(3), 387 - 404, 2003.

[9] P.W. Hemker, G.I. Shishkin and L.P. Shishkina. High-order accurate decomposition of richardson's method for a singularly perturbed elliptic reaction-diffusion equation. Comp. Math. Math. Phys, 44(2), 309 - 316, 2004.

[10] V.L. Kolmogorov and G.I. Shishkin. Numerical methods for singularly perturbed boundary value problems modeling diffusion processes. In: J.J.H. Miller(Ed.), Singular Perturbation Problems in Chemical Physics, Advances in Chemical Physics, John Willey \& Sons, New York, 181 - 362, 1997.

[11] O.A. Ladyzhenskaya, V.A. Solonnikov and N.N. Ural'tseva. Linear and Quasilinear Equations of Parabolic Type. Translations of Mathematical Monographs, 23, Americal Mathematical Society, USA, 1968.

[12] J.J.H. Miller, E. O'Riordan and G.I. Shishkin. Fitted numerical methods for singular perturbation problems. World Scientific, Singapore, 1996.

[13] H.-G. Roos, M. Stynes and L. Tobiska. Numerical Methods for Singularly Perturbed Differential Equations. Convection-Diffusion and Flow Problems. Springer-Verlag, Berlin, 1996.

[14] A.A. Samarskii. The Theory of Difference Schemes. Marcel Dekker, New York, 2001.

[15] G.I. Shishkin. The method of increasing the accuracy of solutions of difference schemes for parabolic equations with a small parameter at the highest derivative. USSR Comput. Maths. Math. Phys., 24(6), 150 - 157, 1984.

[16] G.I. Shishkin. Grid approximation of a singularly perturbed boundary value problem for a quasilinear elliptic equation in the case of complete degeneration. Zh. Vychisl. Mat. Mat. Fiz., 31(12), 1808 - 1825, 1991. (in Russian)

[17] G.I. Shishkin. Grid approximation of a singularly perturbed boundary value problem for quasilinear parabolic equations in the case of complete degeneracy in spatial variables. Sov. J. Numer. Anal. Math. Modelling, 6(3), 243 - 261, 1991.

[18] G.I. Shishkin. Discrete approximation of singularly perturbed elliptic and parabolic equations. Russian Academy of Sciences, Ural Section, Ekaterinburg, 1992. (in Russian)

[19] G.I. Shishkin. Singularly perturbed boundary value problems with concentrated sources and discontinuous initial conditions. Comp. Maths. Math. Phys., 37(4), 417 - 434, 1997.

[20] G.I. Shishkin. Finite-difference approximations of singularly perturbed elliptic equations. Comp. Maths. Math. Phys., 38(12), 1909 - 1921, 1998.

[21] G.I. Shishkin. Grid approximations with an improved rate of convergence for singularly perturbed elliptic equations in domains with characteristic boundaries. Siberian Zh. Vychisl. Mat., 5(1), 71 - 92, 2002. (in Russian)

[22] G.I. Shishkin. Grid approximation of improved convergence order for a singularly perturbed elliptic convection-diffusion equation. In: Proc. of the Steklov Institute of Mathematics. Suppl. 1, $184-202,2003$.

[23] G.I. Shishkin. Grid approximation of parabolic convection-diffusion equations with piecewise smooth initial data. Dokl. Rus. Akad. Nauk., 405(1), $1-4,2005$. (in Russian) 
[24] G.I. Shishkin. Grid approximation of the domain and solution decomposition method with improved convergence rate for singularly perturbed elliptic equations in domains with characteristic boundaries. Comp. Maths. Math. Phys., 45(7), 1155 - 1171, 2005.

[25] G.I. Shishkin and L.P. Shishkina. A higher-order richardson method for a quasilinear singularly perturbed elliptic reaction-diffusion equation. Differential Equations, 41(7), $1030-1039,2005$.

\section{Stiprūs nauji aukštos eilès tikslūs skaitmeniniai metodai singuliariai sutrukdytiems konvekcijos-difuzijos uždaviniams}

\section{G.I. Šiškinas}

Straipsnyje nagrinejjami nedidelio tikslumo $\varepsilon$ - tolygiai konvertuojantys skaitmeniniai metodai, singuliariai sutrikdytiems kraštiniams uždaviniams. Paraboliniam konvekcijos-difuzijos uždaviniui konvergavimo eilè neviršija vienos antrosios, jeigu uždavinio duomenys yra pakankamai glodūs. Tačiau trūkiems pradiniams duomenims eilè yra ne aukštesnè už $2^{-1}$. Šio tipo uždaviniams, naudojant naujai išvestus metodus, darbe sukonstruotos $\varepsilon-$ tolygiai konvertuojančios schemos aukštesniu tikslumu. 